\title{
Ex vivo piperaquine resistance developed rapidly in Plasmodium falciparum isolates in northern Cambodia compared to Thailand
}

Suwanna Chaorattanakawee ${ }^{1,2}$, Chanthap Lon ${ }^{1,3^{*}}$, Krisada Jongsakul $^{1}$, Jariyanart Gawee ${ }^{4}$, Somethy Sok ${ }^{5}$, Siratchana Sundrakes ${ }^{1}$, Nareth Kong ${ }^{6}$, Chatchadaporn Thamnurak', Soklyda Chann ${ }^{3}$, Sorayut Chattrakarn', Chantida Praditpol ${ }^{1}$, Nillawan Buathong ${ }^{1}$, Nichapat Uthaimongkol ${ }^{1}$, Philip Smith' ${ }^{1}$, Narongrid Sirisopana ${ }^{4}$, Rekol Huy ${ }^{6}$, Satharath Prom ${ }^{5}$, Mark M. Fukuda ${ }^{1}$, Delia Bethell ${ }^{1}$, Douglas S. Walsh' ${ }^{1}$, Charlotte Lanteri ${ }^{1,7}$ and David Saunders ${ }^{1}$

\begin{abstract}
Background: The recent dramatic decline in dihydroartemisinin-piperaquine (DHA-PPQ) efficacy in northwestern Cambodia has raised concerns about the rapid spread of piperaquine resistance just as DHA-PPQ is being introduced as first-line therapy in neighbouring countries.
\end{abstract}

Methods: Ex vivo parasite susceptibilities were tracked to determine the rate of progression of DHA, PPQ and mefloquine (MQ) resistance from sentinel sites on the Thai-Cambodian and Thai-Myanmar borders from 2010 to 2015. Immediate ex vivo (IEV) histidine-rich protein 2 (HRP-2) assays were used on fresh patient Plasmodium falciparum isolates to determine drug susceptibility profiles.

Results: IEV HRP-2 assays detected the precipitous emergence of PPQ resistance in Cambodia beginning in 2013 when $40 \%$ of isolates had an $\mathrm{IC}_{90}$ greater than the upper limit of prior years, and this rate doubled to $80 \%$ by 2015 . In contrast, Thai-Myanmar isolates from 2013 to 14 remained PPQ-sensitive, while northeastern Thai isolates appeared to have an intermediate resistance profile. The opposite trend was observed for MQ where Cambodian isolates appeared to have a modest increase in overall sensitivity during the same period, with $I C_{50}$ declining to median levels comparable to those found in Thailand. A significant association between increased PPQ IC ${ }_{50}$ and IC $C_{90}$ among Cambodian isolates with DHA-PPQ treatment failure was observed. Nearly all Cambodian and Thai isolates were deemed artemisinin resistant with a $>1 \%$ survival rate for DHA in the ring-stage assay (RSA), though there was no correlation among isolates to indicate cross-resistance between PPQ and artemisinins.

Conclusions: Clinical DHA-PPQ failures appear to be associated with declines in the long-acting partner drug PPQ, though sensitivity appears to remain largely intact for now in western Thailand. Rapid progression of PPQ resistance associated with DHA-PPQ treatment failures in northern Cambodia limits drugs of choice in this region, and urgently requires alternative therapy. The temporary re-introduction of artesunate AS-MQ is the current response to PPQ resistance in this area, due to inverse $\mathrm{MQ}$ and $\mathrm{PPQ}$ resistance patterns. This will require careful monitoring for re-emergence of $\mathrm{MQ}$ resistance, and possible simultaneous resistance to all three drugs (AS, MQ and PPQ).

Keywords: Malaria, Drug resistance, Piperaquine, Mefloquine, Cambodia, Thailand

*Correspondence: Chanthapl.ca@afrims.org

${ }^{3}$ USAMC-AFRIMS, Phnom Penh, Cambodia

Full list of author information is available at the end of the article 


\section{Background}

Artemisinin combination therapy (ACT) remains the most effective currently available regimen for multidrug-resistant falciparum malaria, and as such is key to reducing the global malaria burden $[1,2]$. While artemisinin-resistant Plasmodium falciparum has emerged in the western provinces of Cambodia, and some neighboring countries in Southeast Asia [3-6], ACT appears to remain clinically effective where partner drug efficacy is preserved. Dihydroartemisinin-piperaquine (DHAPPQ) has been widely adopted in the region as a first-line agent to treat multi-drug resistant falciparum malaria. In 2008, it replaced artesunate-mefloquine (AS-MQ) in select areas of western Cambodia due to AS-MQ treatment failures [7]. In early studies, various PPQ-containing ACT showed excellent safety and tolerability with efficacy of 96-98 \% in Cambodia [8-12], and DHA-PPQ was implemented as the drug of choice countrywide a few years later [13].

Treatment failures were detected soon after, as early as 2010, with PCR-corrected day- 42 failure rate of $25 \%$ in Pailin and $11 \%$ in Pursat Provinces in Cambodia [14]. In 2013, high grade DHA-PPQ failure of $53 \%$ was first reported in Oddar MeanChey province with corresponding increases in $\mathrm{PPQ}$ in vitro $\mathrm{IC}_{50}$ higher than patient plasma PPQ levels during the terminal elimination phase [15-17]. Subsequent reports confirmed rapidly increasing DHA-PPQ failure rates in areas with previously documented artemisinin (ART) resistance $[18,19]$. Despite the rapid loss of PPQ sensitivity, multiple studies detected a simultaneous decline in $p f m d r 1$ copy number amplification and increased in vitro MQ sensitivity among PPQ-resistant isolates $[15,18,20]$. The resurgence in MQ sensitivity suggested that short-term re-introduction MQ-containing therapy or perhaps triple artemisinin combination therapy (TACT) containing both PPQ and MQ might be effective. While approaches are currently being pursued to various degrees by public health authorities and research organizations [18], there is no consensus regarding safety and resistance concerns related to the use of TACT [21].

To address this growing public health crisis, intensive monitoring of drug resistance profiles remains crucial information to determine appropriate alternatives in settings of rapidly emerging multi-drug anti-malarial resistance. In the absence of a known molecular PPQ resistance marker, field-based ex vivo parasite drug susceptibility testing using fresh $P$. falciparum isolates remains a cost-effective, rapid, surveillance tool to track resistance [22]. Here, parasite drug susceptibilities to ART, PPQ and MQ between 2010 and 2015 on both sides of the Thai-Cambodian border, and in southwestern Thailand along the border with Myanmar were reported.

\section{Methods}

Study sites, protocols, and subjects

Between October 2010-April 2015, 342 P. falciparum mono-infections from Preah Vihear (PV) and Oddar MeanChey (OM) provinces in northern Cambodia, Pursaron (PL) village in Srisaket province in northeast Thailand, and Kwai River Christian Hospital (KRCH) in Kanchanaburi province in western Thailand were collected for evaluation for PPQ, MQ and ART susceptibility. All samples were collected from patients with uncomplicated malaria enrolled in five clinical protocols (clinical trials WR1737-NCT01280162; WR1877NCT01849640; WR2017-NCT02052323; WR1576 and WR1917, both in vitro surveillance studies). Figure 1 shows site locations and the number of samples collected in each site per year. All protocols were approved by the Walter Reed Army Institute of Research (WRAIR) Institutional Review Board, and Cambodian National Ethics Committee for Health Research (NECHR), Institute for Development of Human Research Protection (IHRP), Ministry of Public Health, Thailand or Royal Thai Army Institutional Review Board (RTA IRB), respectively. All study subjects provided informed consent prior to participation.

\section{Parasite susceptibility assay to PPQ and MQ}

At the PV, OM and $\mathrm{KRCH}$ sites, blood samples were collected prior to treatment and tested within 2-6 h for ex vivo susceptibility to PPQ and MQ using histidine rich protein-2 (HRP-2) ELISA to measure 50 and $90 \%$ inhibitory concentration ( $\mathrm{IC}_{50}$ and $\mathrm{IC}_{90}$ ) following previously published methods [15]. Briefly, samples with a parasitaemia of $\leq 0.5 \%$ were adjusted to $1.5 \%$ haematocrit in $0.5 \%$ Albumax RPMI 1640 (containing $25 \mathrm{mM}$ HEPES, $25 \mathrm{mM}$ sodium bicarbonate, and $0.1 \mathrm{mg} / \mathrm{mL}$ gentamycin), whereas those with $>0.5 \%$ parasitaemia were diluted to the parasitaemia range of $0.2-0.5 \%$ by adding $50 \%$ haematocrit human $\mathrm{O}+$ red blood cells in $10 \%$ serum-RPMI 1640 and adjusted to a $1.5 \%$ haematocrit in $0.5 \%$ Albumax RPMI 1640 prior to adding to dried drugcoated plates. Parasites were then incubated for $72 \mathrm{~h}$ at $37{ }^{\circ} \mathrm{C}$ in a candle jar, after which plates were frozen and later thawed for analysis of growth inhibition using the HRP-2 ELISA. Due to limited amount of patient blood, ex vivo assay was performed as a singlet experiment. At the PL site, patient blood samples were cryopreserved in a glycerol mixed solution, then shipped to the AFRIMS laboratory in Bangkok to establish in vitro culture, and then tested for drug susceptibility as described previously [22]. Briefly, synchronized cultures with $\geq 90 \%$ ring forms were diluted to $0.5 \%$ parasitaemia with $1.5 \%$ haematocrit in $0.5 \%$ Albumax RPMI 1640, and transferred to dried drug-coated plates. Plates were incubated 


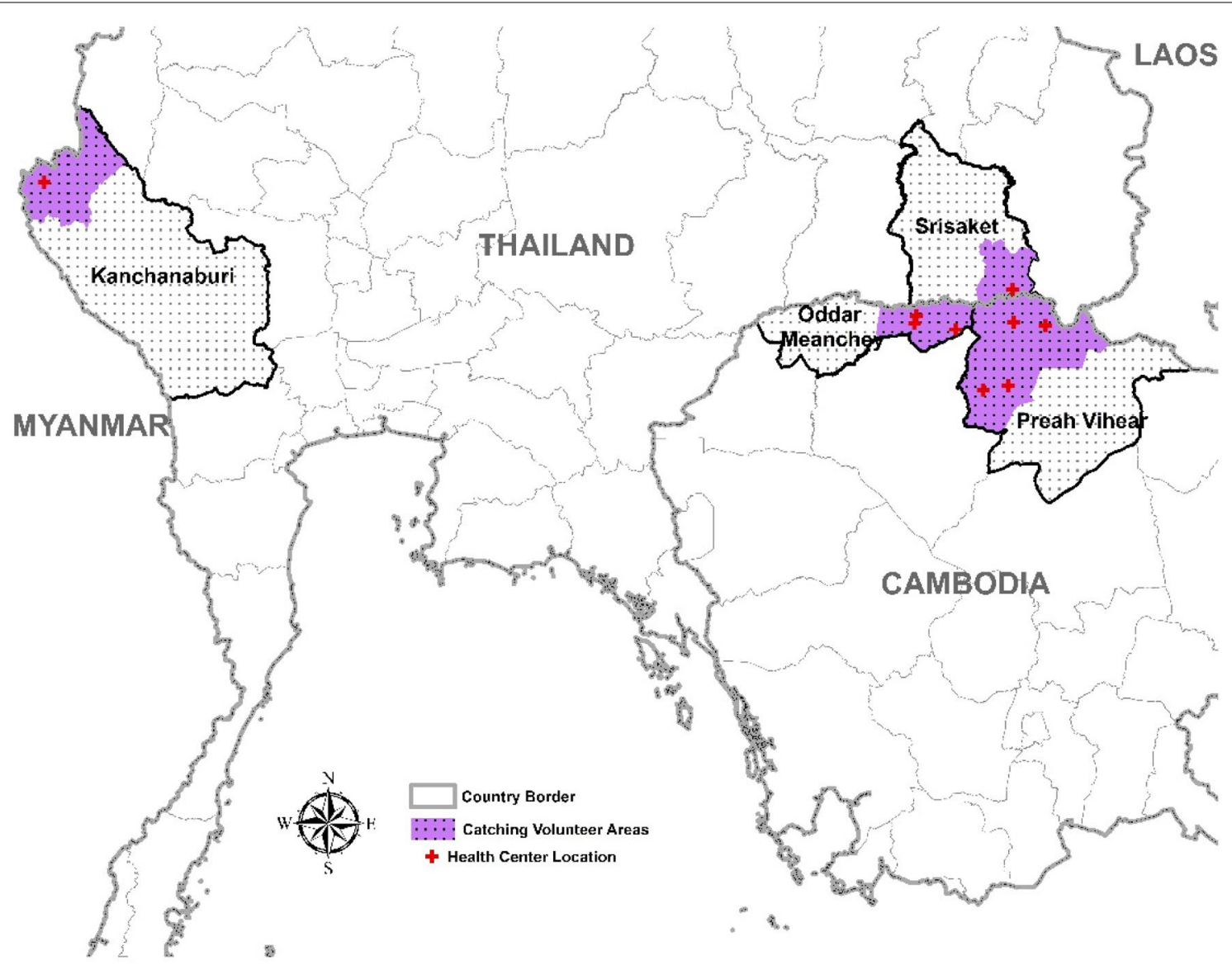

\begin{tabular}{|c|c|c|c|c|c|c|c|c|}
\hline \multirow{2}{*}{ Country } & $\begin{array}{c}\text { Collection } \\
\text { Site }\end{array}$ & \multicolumn{6}{|c|}{ Number of samples per year } & \multirow{2}{*}{ Total } \\
\cline { 3 - 10 } & 2010 & 2011 & 2012 & 2013 & 2014 & 2015 & \\
\hline \multirow{2}{*}{ Cambodia } & PV & - & 39 & - & - & - & - & 39 \\
\cline { 2 - 10 } & $\mathrm{OM}$ & 20 & 7 & 9 & 98 & 85 & 35 & 254 \\
\hline \multirow{2}{*}{ Thailand } & $\mathrm{KRCH}$ & - & - & - & 8 & 14 & - & 22 \\
\cline { 2 - 9 } & $\mathrm{PL}$ & - & - & - & 22 & 5 & - & $27^{*}$ \\
\hline
\end{tabular}

Fig. 1 Sentinel sites for Plasmodium falciparum isolate collection 2010-2015. The map shows location of isolate collection sites: Preah Vihear (PV) and Oddar MeanChey (OM) provinces in Cambodia, the Kwai River Christian Hospital (KRCH) in Kanchanaburi province, and Pursaron (PL) village in Srisaket province, Thailand. Dot patterns denote collection provinces; colour shading indicates regional district areas, respectively, with individual health centres indicated by a plus sign. The Table includes collected isolates per site. Asterisk all samples from PV, OM and KRCH were tested fresh using the IEV assay. The 27 isolates collected from PL were initially cryopreserved; only 14 could be recovered from cryopreservation and maintained in culture to be tested for in vitro drug susceptibility

at $37{ }^{\circ} \mathrm{C}$ with $5 \% \mathrm{CO}_{2}, 5 \% \mathrm{O}_{2}$ and $90 \% \mathrm{~N}_{2}$ for $72 \mathrm{~h}$. Assay was done for 3-5 replicates experiments and averaged values were reported. Parasite growth inhibition was assessed using the HRP-2 ELISA. As an assay benchmark, the culture of reference $P$. falciparum W2 were tested for in vitro drug susceptibility as described above. All test drugs were provided by the WRAIR (Silver Spring, MD,
USA). MQ and PPQ were dissolved in $70 \%$ ethanol and $0.5 \%$ lactic acid in distilled water to make $1 \mathrm{mg} / \mathrm{mL}$ stock solutions, respectively. Details for preparing drug coated plates was described previously [23].

Parasite growth after $72 \mathrm{~h}$ was assessed by HRP-2 ELISA. HRP-2 optical density (OD) readings were plotted against drug concentrations, $\mathrm{IC}_{50} \mathrm{~s}$ and $\mathrm{IC}_{90} \mathrm{~s}$ were 
estimated by non-linear regression analysis using the Graph-Pad Prism version 6.0 (GraphPad Software, Inc.-, San Diego, CA, USA). PPQ and MQ concentrations in the assay ranged from 0.9 to $674 \mathrm{nM}$ and 0.3 to $200 \mathrm{nM}$, respectively, as well as a drug-free control to assess normal parasite growth. Starting in 2013, there were evidences that field isolates were able to grow in extremely high PPQ concentrations, requiring maximum concentrations as high as 53,905 $\mathrm{nM}$ in order to obtain interpretable drug susceptibility curves. This was far higher than the standard maximum PPQ level used (674 nM). Therefore, since 2014, a serial dilution range to achieve higher maximum PPQ concentrations (3.4-53,905 nM) was used in addition to the standard dilutions to ensure accurate inhibitory concentrations could be determined. In an attempt to estimate PPQ $\mathrm{IC}_{50}$ and $\mathrm{IC}_{90}$ of reduced susceptible isolates from 2013 tested on standard PPQ dilution range ( 0.9 to $674 \mathrm{nM})$, reanalysis was done to fit 'zero-growth' OD values at the extrapolated PPQ concentration of 53,905 $\mathrm{nM}$ to better estimate accurate doseresponse curves [24].

\section{Ring-stage survival assay (RSA) for ART susceptibility}

A portion of Cambodian and northeast Thai isolates was tested for sensitivity to ARTs using a ring-stage survival assay (RSA). Cambodian samples were tested for ex vivo RSA within 2-6 h of phlebotomy, without prior culture adaptation or parasite synchronization steps following published methods [25]. Briefly, samples with parasitaemias of $\leq 1 \%$ were adjusted to $2 \%$ haematocrit in culture media (0.5 \% Albumax RPMI 1640 with $2.5 \%$ AB serum), and cultured in a 48-well microplate with $700 \mathrm{nM}$ DHA and $0.1 \%$ DMSO in separate wells for growth control. Samples with $>1 \%$ parasitaemia were diluted to a parasitaemia range of $0.5-1 \%$ by adding $50 \%$ haematocrit human O+ red blood cells in $10 \%$ serum-RPMI 1640 prior to adjusting to a $2 \%$ haematocrit. The culture plate was then incubated for $6 \mathrm{~h}$ at $37^{\circ} \mathrm{C}$ in a candle jar, after which culture medium was discarded. Cells were then washed, re-suspended in drug-free medium, and cultured for $66 \mathrm{~h}$. Susceptibility to DHA was assessed microscopically on thin films by estimating the percentage of viable parasites, relative to control (\% survival rate).

Similar methods were performed on Thai isolates, but instead of ex vivo testing on clinical samples, in vitro parasite culture was established for each sample and an in vitro $\mathrm{RSA}^{0-3 \mathrm{~h}}$ was performed on $0-3$-h post-invasion rings obtained from culture-adapted parasites following published methods [25]. Briefly, parasites cultures were synchronized utilizing $5 \%$ D-sorbitol and $75 \%$ Percoll to obtain 0 to 3 -h post-invasion rings which were adjusted to $0.5-1 \%$ parasitaemia with a $2 \%$ haematocrit in culture media. The following processes were performed as an ex vivo RSA, but parasites were cultured with mixed gas $\left(5 \% \mathrm{CO}_{2}, 5 \% \mathrm{O}_{2}\right.$ and $\left.90 \% \mathrm{~N}_{2}\right)$, instead of using a candle jar. As quality controls for the assay, the RSA ${ }^{0-3 h}$ was also performed on $P$. falciparum reference clones W2, IPC4884 and IPC-5202 (Malaria Research \& Reference Reagent Resource, Manassas, Vermont, USA) to ensure an acceptable range of \% survival rate was attained against these reference clones.

\section{Statistical analysis}

Statistical analysis was performed using Graph-Pad Prism version 6.0 (GraphPad Software, Inc, San Diego, CA, USA). Parasite drug susceptibilities were expressed as median $\mathrm{IC}_{50} \mathrm{~s}$ and $\mathrm{IC}_{90} \mathrm{~s}$ for all isolates. Differences in susceptibility between groups were determined using non-parametric Mann-Whitney or Kruskal-Wallis tests and multiple comparison tests as appropriate. Correlations between PPQ ICs and \% survival rate in the presence of DHA were analyzed by calculating Spearman's correlation coefficient. In order to determine the PPQ susceptibility baseline, Grub's test was used for outlier analysis of IC values attained from Cambodian isolates from 2010 to 2012 where no evidence of PPQ resistance was detected.

\section{Results}

HRP-2 dose response curves of Plasmodium falciparum isolates reveals severe ex vivo $P P Q$ resistance

In 2013, evidence of severe ex vivo PPQ resistance not observed in prior years was detected in northern Cambodia when parasite isolates survived exposure at maximum PPQ levels $(675 \mathrm{nM})$ normally employed in the ex vivo HRP-2 assay [24]. Since 2014, a serial dilution range to achieve higher maximum PPQ concentrations (3.4$53,905 \mathrm{nM}$ ) was used in addition to the standard dilutions to ensure accurate inhibitory concentrations could be determined. Figure 2 shows dose response curves for isolates with a range of susceptibilities based on standard PPQ dilutions (0.9-674.8 $\mathrm{nM}$ ) compared to the higher concentration range (3.4-53,905 nM). For PPQ sensitive isolates, sigmoidal curves and reliable analysis were attained from both dilution ranges (Fig. 2a). However, reduced susceptibility isolates were able to grow in the maximum standard PPQ level $(675 \mathrm{nM})$ preventing determination of accurate growth inhibition curves (Fig. 2b, c). Similar to a previous report [26], anomalous curves due to paradoxical parasite growth at high drug concentrations were observed in reduced susceptibility isolates. However, increasing maximum PPQ concentration to $53,905 \mathrm{nM}$ conferred $100 \%$ growth inhibition, attaining reliable dose-response curves. In an attempt 


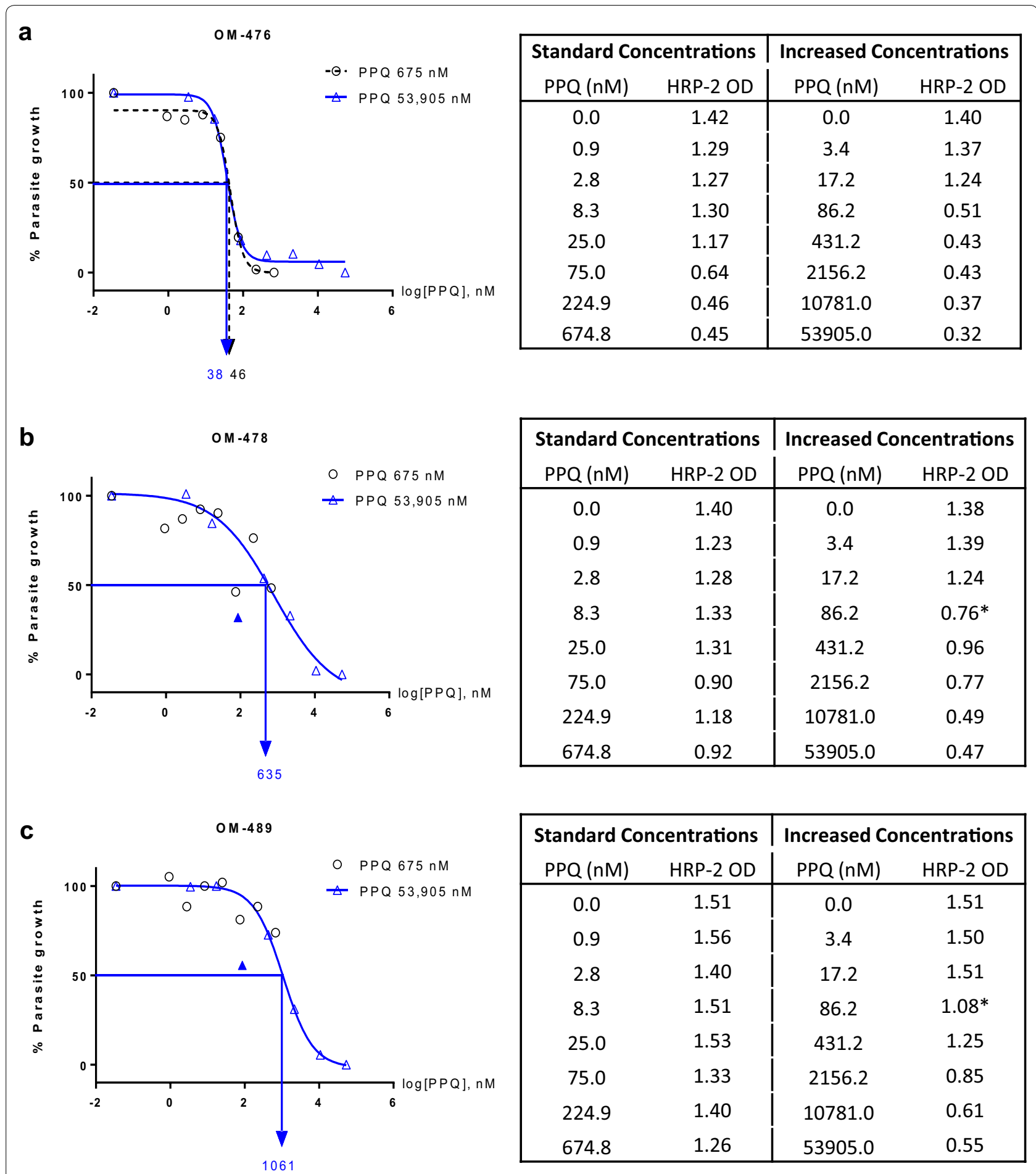

Fig. 2 Representative dose response curves for Plasmodium falciparum isolates with severely reduced PPQ sensitivity. PPQ dose-response curves of P. falciparum isolates with a range of susceptibility. $\mathbf{a}$ is a PPQ-sensitive isolate (OM-476), while $\mathbf{b}$ and $\mathbf{c}$ are PPQ-resistant isolates (OM-478, OM-489). $\%$ parasite growth was plotted against log [PPQ concentration] using the standard PPQ concentration range from 0 to $674.8 \mathrm{nM}$ (black) and an increased concentration range from 0 to $53,905 \mathrm{nM}$ (b/ue), with $\mathrm{IC}_{50}$ for each indicated. Filled symbols represent outlier values excluded from curve fitting. Tables present HRP-2 OD values attained for individual isolates at each concentration range, while asterisk indicates values excluded prior to curve fitting 
to estimate PPQ $\mathrm{IC}_{50}$ and $\mathrm{IC}_{90}$ in these isolates, dose response curves were interpolated by excluding outliers to yield best-fit models (Fig. 2b, c).

\section{Plasmodium falciparum susceptibility to PPQ and MQ from 2010 to 2015}

Of 315 P. falciparum isolates tested using the ex vivo HRP-2 assay, IC results were evaluable in 222 isolates for PPQ and 230 for MQ. The in vitro assay was successful for all 14 isolates recovered among 27 cryopreserved samples. Figure $3 \mathrm{a}$ and $\mathrm{b}$ show PPQ $\mathrm{IC}_{50}$ and $\mathrm{IC}_{90}$ for $P$. falciparum isolates collected from Cambodia and Thailand from 2010 to 2015. Relative to a PPQ-sensitive W2 reference clone, Cambodian isolates from 2010 to 2012 were sensitive to PPQ with median $\mathrm{IC}_{50}$ and $\mathrm{IC}_{90}$ of 17-22 $\mathrm{nM}$, and 41-60 $\mathrm{nM}$, respectively. The emergence of PPQ resistance was detected in OM, Cambodia beginning in 2013, with parasite isolates surviving exposure to maximum tested PPQ levels $(674 \mathrm{nM})$ in the ex vivo assay, with 18 and $40 \%$ of isolates exceeding prior year baselines $\left(\mathrm{IC}_{50}>69 \mathrm{nM}\right.$ and $\left.\mathrm{IC}_{90}>160 \mathrm{nM}\right)$. More than half of isolates with elevated $\mathrm{IC}_{90}$ still had an $\mathrm{IC}_{50}$ below prior year baselines. PPQ resistance worsened in 20142015 with $70-80 \%$ of isolates having $\mathrm{IC}_{90}$ and $\mathrm{IC}_{50}$ above prior baseline levels, in some cases by a thousand-fold over prior years.

In contrast, western Thai isolates from the same time period (2013-14) along the border with Myanmar were PPQ-sensitive with a median $\mathrm{IC}_{90}$ of $56.7 \mathrm{nM}$ comparable to the 2010-12 Cambodian $\mathrm{IC}_{90}$ of $60.4 \mathrm{nM}$. Northeastern Thai isolates from 2013 to 14 collected from PL, roughly $100 \mathrm{~km}$ from Anlong Veng on the Thai-Cambodian border had intermediate PPQ sensitivity with a median $\mathrm{IC}_{90}$ of $238.8 \mathrm{nM}$. Kruskal-Wallis and multiple comparison analysis revealed significant elevations in median Cambodian isolate PPQ $\mathrm{IC}_{50}$ and $\mathrm{IC}_{90}$ values from 2013 to 2015, compared to prior years, as well as western Thai isolates from the same time period $(P<0.05)$. Modestly increased $\mathrm{IC}_{50 / 90}$ values for cryopreserved, northeastern Thai isolates were not statistically different from ex vivo isolates from western Thailand and Cambodia. The opposite trend was observed for MQ susceptibility (Fig. 3c) where Cambodian isolates developed increased susceptibility to MQ from 2013 to 2015, with lower median $\mathrm{IC}_{50}$ compared to prior years and to western Thai isolates (Kruskal-Wallis test, $P=0.008$ ).

\section{Association of reduced PPQ susceptibility with clinical DHA-PPQ treatment failure}

Emergence of PPQ resistance associated with DHA-PPQ treatment failure was identified in clinical studies conducted in Cambodia from 2010 to 13 [17]. Plasmodium falciparum isolates from those studies capable of ex vivo growth in the presence of $675 \mathrm{nM} P \mathrm{PQ}$ were more common in subjects suffering $P$. falciparum recrudescence within 42 days (43\%) than those without recrudescence $\left(8 \% ; \chi^{2}=16.68 ; P<0.001\right)$. Isolates from subjects with $P$. falciparum recrudescence had significantly increased $\mathrm{IC}_{50}$ and $\mathrm{IC}_{90}$ compared to those without recrudescence, most notably for $\mathrm{IC}_{90}$, which was three-fold higher than in those without recrudescence (median $\mathrm{IC}_{50} 38$ vs $26 \mathrm{nM}, P$ value of Mann-Whitney $\mathrm{U}$ test $=0.009$; median $\mathrm{IC}_{90}$ of 201 vs $77 \mathrm{nM}, P<0.001$ ). Elevated $\mathrm{IC}_{50}$ above prior baselines was more common in isolates from recrudescent cases $(9 / 33=27 \%)$, but was found in only $8 \%$ of those without recrudescence $\left(4 / 48 ; \chi^{2}=5.21\right.$; $P=0.023)$. Similar results were found for $\mathrm{IC}_{90}$ with values above prior years in 55 and $23 \%$ of isolates from recrudescent and non-recrudescent cases, respectively $\left(\chi^{2}=8.51 ; P=0.004\right)$.

\section{Drug susceptibility profiles do not suggest cross-resistance between PPQ and ARTs}

To examine possible cross-resistance between PPQ and AS, 85 Cambodian isolates from 2014 to 2015 and 14 northeastern Thai isolates from 2013 to 2014 were tested in the RSA to measure \% survival rate against DHA, and their association with PPQ $\mathrm{IC}_{50}$ and $\mathrm{IC}_{90}$ was assessed. Survival rates for 72 evaluable Cambodian isolates ranged from 0 to $100 \%$ with a median of 7.7 and IQR of 2.0-16.4, while reference clone survival was as expected with medians of $0.3,2.7$, and $79.1 \%$ for ART-sensitive W2, ART-resistant IPC-4884 and IPC-5202, respectively (Fig. 4). Based on an ART resistance cut-off above $1 \%$ survival, only $22 \%$ of Cambodian isolates (16/72) were sensitive to ARTs, while $78 \%$ (56/72) were deemed resistant. No significant differences in the proportion of resistant isolates between $2014(47 / 54=87 \%)$ and $2015(13 / 18=72 \%)$ were found $\left(x^{2}=2.13, P>0.05\right)$, although there was a decrease in median survival rate for 2015 isolates (9.6), compared to those from 2014 (4.8), Mann-Whitney test, $P=0.017$. Median survival rate for western Thai isolates was 24.0 with an IQR of 4.7-48.6, nearly all (13/14) were resistant to ARTs with survival rate above $1 \%$. Due to limited data available for Thailand, the change in ART resistance over time was not evaluated.

There was not a significant correlation between \% survival rate in the presence of ARTs and PPQ $\mathrm{IC}_{50}$ or $\mathrm{IC}_{90}$ (Spearman $\rho<0.1, P>0.05$ ). ART-resistant Cambodian isolates (survival rate $>1 \%$ ) had higher PPQ ICs than sensitive isolates (survival rate $\leq 1 \%$ ), but differences were not statistically significant with $\mathrm{IC}_{50}$ of $448 \mathrm{nM}$ for resistant isolates vs $268 \mathrm{nM}$ for sensitive ( $P$ value for Mann-Whitney U test $=0.99$ ), and $\mathrm{IC}_{90} \mathrm{~s}$ of $9175 \mathrm{vs}$ $4489 \mathrm{nM}$, respectively $(P=0.84)$. 


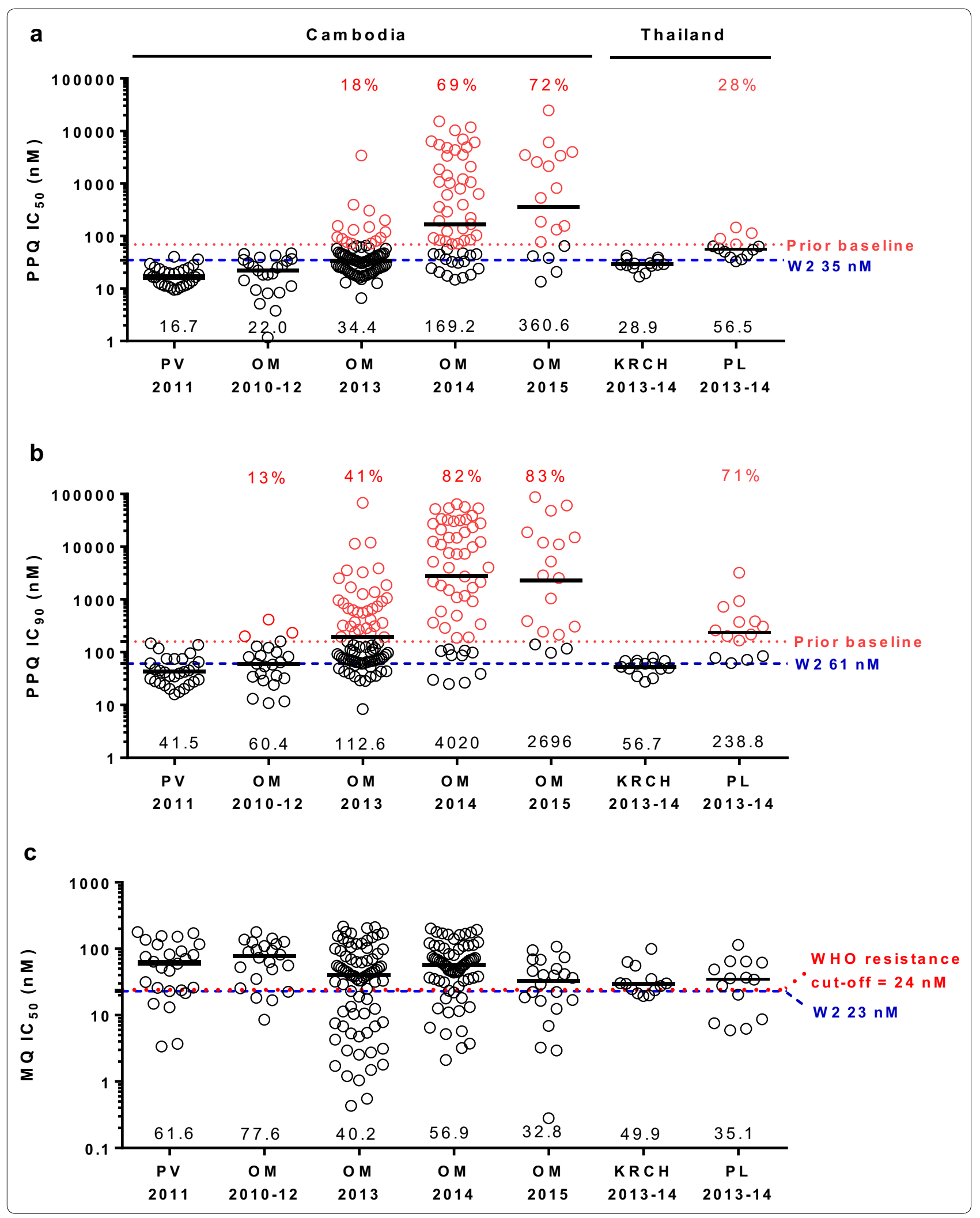


(See figure on previous page.)

Fig. 3 PPQ and MQ susceptibility of Cambodian and Thai isolates collected from 2010 to 2015. Panels $\mathbf{a}$ and $\mathbf{b}$, respectively, present ex vivo $P$. falciparum $I C_{50}$ and IC $C_{90}$ for $P P Q$, while $M Q I C_{50}$ is shown in panel $\mathbf{c}$. Site/year where samples were collected are indicated on the $X$-axis—Preah Vihear (PV), and Oddar MeanChey (OM) provinces of Cambodia, and Kwai River Christian Hospital (KRCH) in Kanchanaburi Province and Pursaron (PL) in Srisaket Province, Thailand, with median values of $I_{50}$ or $I C_{90}$ denoted for each site/year with a black bar and indicated on X-axis. Red dotted lines indicate the PPQ susceptibility baseline of Cambodian isolates from 2010-2012 (a, b) and WHO values for suspected MQ resistance (c). The W2 susceptibility value is indicated by blue dashed lines. Isolates above prior year baseline for PPQ $\left(I_{50}>69 \mathrm{nM}, I C_{90}>160 \mathrm{nM}\right)$ are in red, with the proportion isolates above baseline indicated

\section{Discussion}

Monitoring ex vivo drug susceptibility of $P$. falciparum revealed rapid progression of PPQ resistance in northern Cambodia between 2013 and 2015, following reports of severe clinically significant resistance in 2013 [15, 18], while western Thai isolates collected from the same time period remained sensitive to PPQ corresponding with a recent report of $94 \%$ efficacy of DHA-PPQ in this region [5]. The northeastern Thai isolates showed modest worsening of PPQ resistance given their close proximity to the Cambodian parasite populations studied (within $100 \mathrm{~km}$ ). Increased PPQ IC $\mathrm{IC}_{50}$ and $\mathrm{IC}_{90}$ associated with recrudescence in DHA-PPQ clinical studies from 2010 to 2013 corresponded with the first reports of DHA-PPQ clinical treatment failures in Cambodia [18, 19, 26]. The findings enforce those of recent studies, indicating the rapid expansion of PPQ-resistant parasites in Cambodia, as well as early signs that piperaquine resistance is emerging in Thailand. Given rapid emergence in Cambodia, and the fact that DHA-piperaquine has only recently been introduced on a large scale, the possibility for rapid worsening of resistance in Thailand is high.
Limited treatment to choose from there is an urgent need for alternative therapies in the region. Whether the rapid expansion of PPQ resistance is due to increased transmission potential of PPQ-resistant isolates as observed for ART-resistant parasites [6] or slow-clearing parasites following DHA-PPQ treatment [27], requires further investigation.

The majority of both Cambodian and Thai isolates were resistant to ARTs in a RSA, consistent with a high prevalence of mutations in the propeller domain of the P. falciparum kelch13 associated with ART resistance in nearly $100 \%$ of isolates [15]. This supports emerging PPQ resistance on an ART resistance background given the high rate of clinical DHA-PPQ failures [18, 26], although there was no clear cross-resistance between PPQ and the ARTs, in part given the very high rate of ex vivo ART resistance. Similar to previous reports $[15,18,26]$, the opposite trend was observed for MQ with increased ex vivo sensitivity noted in Cambodian isolates from 2013-2015. Studies also revealed the association of DHAPPQ treatment failure with single copy of $P$. falciparum multi-drug resistance gene ( $p f m d r 1)$ and decreased $\mathrm{MQ}$

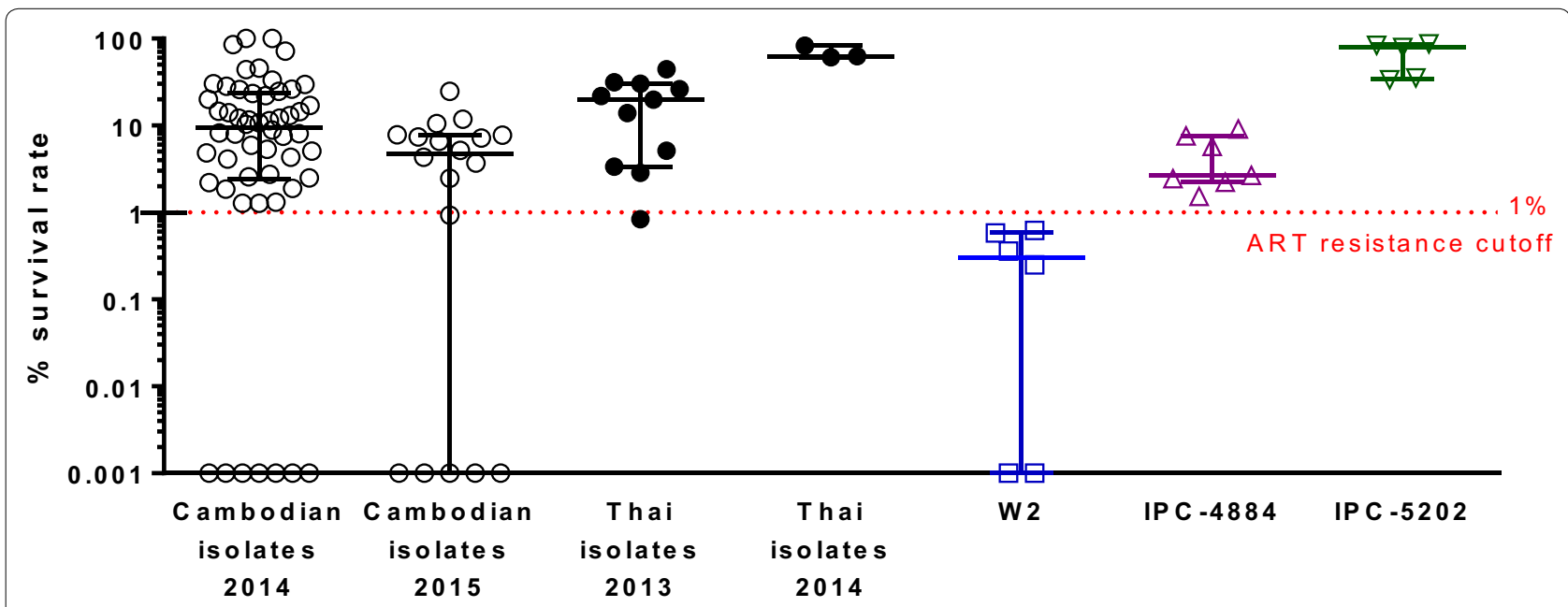

Fig. 4 Ring-stage survival assay (RSA) for artemisinin susceptibility of Plasmodium falciparum isolates and reference clones. Black unfilled and filled circles represent \% survival rate in the ex vivo RSA for Cambodian isolates from Oddar MeanChey (OM) province and in vitro ${ }^{0-3 \mathrm{~h}}$ RSA for Thai isolates from Pursaron (PL) village in Srisaket province, respectively. Blue squares denote values of ART-sensitive (W2) clones while triangles indicate ART-resistant clones (IPC-4884-purple and IPC-5202-green) obtained from 5 to 7 independent in vitro ${ }^{0-3 \mathrm{~h}}$ RSA experiments. Bars represent median and interquartile range. Zero values of \% survival rate were plotted as $0.001 \%$ in logarithmic scale 
$\mathrm{IC}_{50}$. The concomitant increase in MQ sensitivity coupled with worsening PPQ resistance could be explained by declining PPQ susceptibility within parasite population, being genetically distinct from those of MQ resistance. A recent population genetics study revealed emergence of PPQ resistance related to clonal expansion of MQ-sensitive parasites with a single $p f m d r 1$ copy (Parobek et al. pers. comm.)

Ex vivo parasite susceptibility to $\mathrm{PPQ}$, particularly the $\mathrm{IC}_{90}$, appears to be a sensitive, field-expedient and relatively cost-effective marker to detect emerging PPQ resistance at sentinel sites in high-risk areas. Surveillance data first revealed the occurrence of PPQ-resistant parasites in Cambodia in 2010, a year before the clinical failure rate of DHA-PPQ began to increase. Although isolates from 2010 largely remained in the $\mathrm{IC}_{50}$ susceptible range, elevated $\mathrm{IC}_{90}$ was detected in three isolates, two of which were in patients who failed DHA-PPQ treatment [28]. In 2013 when clinical failure for DHA-PPQ was prevalent, $\mathrm{IC}_{90}$ was elevated in $40 \%$ of isolates though more than half still had $\mathrm{IC}_{50}$ in the sensitive range. A rapid progression of resistance was detected in years following with rapid rises in both $\mathrm{IC}_{90}$ and $\mathrm{IC}_{50}$ for most isolates. Decreased parasite susceptibility to PPQ was associated with DHA-PPQ treatment failure, notably for $\mathrm{IC}_{90}$, which was three-fold higher in subjects failing treatment. $\mathrm{IC}_{90}$ appeared to better elucidate shifts in resistance patterns over time and their association with treatment outcomes compared to $\mathrm{IC}_{50}$. Anomalous curves observed with PPQ-resistant isolates in the HRP-2 assay required PPQ concentrations up to $53,905 \mathrm{nM}$ (>25 times higher than the maximum concentration used by Duru et al. [26] before $100 \%$ growth inhibition producing an interpretable, sigmoidal, dose-response curve could be achieved (Fig. 2). Similar to findings with HRP-2, a recent study reported poor performance of the standard hypoxanthine uptake assay due to the high frequency of non-interpretable curves observed with PPQ-resistant isolates. The anomalous curves produced suggested a paradoxical increase in parasite growth at PPQ concentrations above 100-200 nM, reflecting a resistance mechanism induced at physiological concentrations $(200 \mathrm{nM})$ observed in the blood of patients treated with DHA-PPQ [26, 29]. Evidence of paradoxical growth at high concentrations of drugs was previously described in non-ART drug assays [30]. This was suggested to reflect biological properties of drugs, including mechanism of action. Amendment of the lower constraint of the sigmoid model was suggested to provide a more accurate measurement. However, this is not the case for the paradoxical growth seen among resistant isolates in the PPQ assay demonstrated here, or in another recent report [26], and may represent a phenomenon of PPQ-resistant isolates.
To compensate for these challenges for interpretation, a novel in vitro assay for PPQ resistance, the PPQ survival assay (PSA), was designed to mimic in vivo exposure of parasites to a pharmacologically relevant dose of PPQ $(200 \mathrm{nM})$ for $48 \mathrm{~h}$ with the \% parasite survival rate measured at $24 \mathrm{~h}$ after drug exposure [18, 21, 26]. A 48-h drug incubation period allows adequate PPQ exposure for all parasite stages from 0 -h rings to 48 -h schizonts, given PPQs having long clinical half-life ( $\sim 9$ days) $[26,29]$. As a result, the assay does not assess parasite susceptibility to PPQ at a specific parasite stage, but rather the entire life cycle. PSA was demonstrated to be a useful tool to differentiate PPQ resistance among individual fresh isolates, and the \% survival rate was found to correlate with DHAPPQ treatment outcome. Future investigations employing this method are likely to further elucidate emerging piperaquine resistance, although the requirement for multiple individual microscopy readings on each sample limits assay throughput to some degree. The choice of method (PSA, HRP-2, etc.) should be based on available resources and experience. In our view, consistency of application, reproducibility and interpretability of results across time and place are far more important than the technique selected.

The limited sample size for Thai isolates, as well as lack of more recently collected samples are important limitations of the current study. This was mitigated by the sequential approach employed at the sentinel sites, increasing the degree of confidence that median values presented here were reflective of relative drug sensitivities at the community level. Isolates from the ThaiMyanmar border remained highly sensitive to PPQ despite ART resistance comparable to the levels seen in Cambodia, as well as modest MQ resistance. Samples were collected before the widespread use of PPQ in Thailand when AS-MQ remained the first-line drug. While there has clearly been rapid expansion of resistance in northern Cambodia, there appears to be only limited spread across the Thai border, with isolates from nearby PL, Thailand having only moderate declines in PPQ susceptibility. This may be due in part to a lower burden of disease in northeastern Thailand permitting more stringent control measures, and anti-malarial drug administration limited to well-resourced government public health clinics. Regardless, ongoing monitoring is imperative in this region.

Several reports have suggested that ART resistance could promote development of resistance to $\mathrm{PPQ}$, as ART-resistant isolates surviving in the presence of ACT are more likely to spontaneously develop resistance to partner drugs $[18,21]$. The association between PPQ resistance and the K13 mutation likely reflects selection of PPQ-resistant parasites as those initially resistant to 
ARTs remain viable well after ACT treatment has been completed. This has been supported by apparent correlations between increasing DHA-PPQ failure rates on a background of ART-resistant isolates with K13 mutations [17-19]. A recent study reported that all PPQ-resistant isolates carried the K13 mutation, although direct correlation between PPQ and ART susceptibility was not clear as PPQ-sensitive isolates also carried K13 mutation [26]. This may be due to the high prevalence of K13 in the parasite populations studied, and limits potential analysis within the present dataset. Cutoff $\mathrm{IC}_{50}$ or $\mathrm{IC}_{90}$ values for PPQ resistance have yet to be defined, we have not made an effort here to compare \% survival rate to ART between PPQ 'resistant' and 'sensitive' isolates. We performed a Spearman correlation analysis between \% survival rate and PPQ $\mathrm{IC}_{50}$ or $\mathrm{IC}_{90}$ and also compared PPQ $\mathrm{IC}_{50} / \mathrm{IC}_{90}$ between ART-sensitive (RSA $\leq 1 \%)$ and resistant isolates (RSA > $1 \%$ ). A significant correlation between ART and PPQ resistance was not found (see Additional file 1).

Making use of these data for public health purposes presents important challenges, although there does appear to be a key opportunity in the inverse resistance pattern between PPQ and MQ. PPQ resistance appears to be developing in MQ-sensitive parasites with singlecopy Pfmdr-1 and low MQ $\mathrm{IC}_{50}$, as PPQ-resistant isolates in a recent study (PSA survival rates $\geq 10 \%$ ) all had a single $P f m d r-1$ copy and were largely MQ sensitive [26]. Correspondingly, a parasite population genetics study identified the development of resistance to PPQ and MQ in genetically distinct parasite populations (Parobek et al. pers. comm.). In the interim, the national control programme in Cambodia has reverted to the re-introduction of AS-MQ ACT in selected areas of PPQ resistance, and previous studies have observed excellent cure rates using AS-MQ as rescue therapy for DHA-PPQ treatment failures [17, 28]. Recent investigations have proposed triple-drug therapy such as simultaneous AS, MQ and PPQ [18], although the safety of such combinations has not been studied. Further, the possibility of inducing simultaneous resistance to all three drugs amidst rising resistance to the individual components would argue for preserving the advantages afforded by inverse MQ and PPQ resistance patterns by rotating two drug ACT combinations sequentially, combined with inpatient followup of all cases to ensure compliance [21]. Doing so in a carefully coordinated fashion on a biannual basis would permit adequate time to restore at least partial partner drug sensitivity, while holding the current secondline therapy in reserve for clinical treatment failures. Atovaquone-proguanil used as part of public health containment activities in Cambodia may prove to be a useful albeit costly third-line agent, as isolates from the same areas of PPQ resistance in northern Cambodia reported here were recently shown to remain highly susceptible to atovaquone (ATQ) in vitro without evidence of pre-treatment mutations in the cytochrome b gene codon 268 marker for ATQ resistance [24]. However, this liability is well known, with several studies reporting rapid development of single point mutations in cytochrome b conferring ATQ resistance during treatment [31-33]. Neither approach will adequately address the current high-grade ART resistance in the region, although there is hope they may extend the life of current ACT until novel compounds in development, such as the spiroindolones [34] and synthetic ozonides [35], become available.

\section{Conclusions}

The study revealed the rapid expansion of PPQ-resistant parasites in northern Cambodia during 2013-2015, while Thai isolates remained sensitive to PPQ, or showed modest worsening of resistance for those in close proximity to the Cambodian study sites. The opposite trend was observed for MQ with increased ex vivo sensitivity noted in Cambodia. Rapid progression of PPQ resistance associated with the treatment failures of the latest ACT regimen, DHA-PPQ, in northern Cambodia. The findings enforce those of recent studies, indicating the rapid expansion of PPQ-resistant parasites in Cambodia, as well as early signs that piperaquine resistance is emerging in Thailand. Limited drugs of choice in this region highlights the demand alternative therapies. While the re-introduction of AS-MQ ACT remained a treatment choice for PPQ-resistance areas at time of writing, careful monitoring for re-emergence of MQ resistance and/ or simultaneous resistance to all three drugs (AS, MQ and PPQ), as well as of the spread of PPQ resistance to nearby countries, remain crucial to address the resistance crisis.

\section{Additional file}

Additional file 1. Artemisinin and piperaquine susceptibility profiles do not suggest cross resistance.

\begin{abstract}
Abbreviations
AS: artesunate; MQ: mefloquine; PPQ: piperaquine; IEV: immediate ex vivo; HRP-2: histidine-rich protein 2; ELISA: enzyme-linked immunosorbent assay; $I_{50}: 50 \%$ inhibitory concentration; $I_{90}: 90 \%$ inhibitory concentration; RSA: ring-stage survival assay; PSA: piperaquine survival assay; ACT: artemisinin combination therapy; PCR: polymerase chain reaction; TACT: triple artemisinin combination therapy; PV: Preah Vihear; OM: Oddar Meanchey; PL: pursaron; KRCH: Kwai River Christian Hospital; OD: optical density; IQR: interquartile range; ART: artemisinin; Pfmdr 1: P. falciparum multi-drug resistance gene; ATQ: atovaquone; WHO: World Health Organization.
\end{abstract}

\section{Authors' contributions}

Study design Cambodia: SC, CL, DS, SP, and CL; study design Thailand: KJ, NU, MMF, JG, NS, DS, DB, and DSW; data collection: all; data analysis and interpretation: SC, DS, SSundrakes, KJ, and CLon; wrote manuscript: SC, DS, KJ, and CL; All authors read and approved the final manuscript. 


\begin{abstract}
Author details
${ }^{1}$ US Army Medical Component-Armed Forces Research Institute of Medical Sciences (USAMC-AFRIMS), Bangkok, Thailand. ${ }^{2}$ Department of Parasitology and Entomology, Faculty of Public Health, Mahidol University, Bangkok, Thailand. ${ }^{3}$ USAMC-AFRIMS, Phnom Penh, Cambodia. ${ }^{4}$ Royal Thai Army, Bangkok, Thailand. ${ }^{5}$ Royal Cambodian Armed Forces, Phnom Penh, Cambodia. ${ }^{6}$ National Center for Parasitology, Entomology and Malaria Control, Phnom Penh, Cambodia. ${ }^{7}$ Present Address: Department of Pathology and Area Laboratory Services, Microbiology Section, Brooke Army Medical Center, San Antonio, TX, USA.
\end{abstract}

\section{Acknowledgements}

We are grateful to the AFRIMS, Cambodian and Thai clinical and laboratory field teams for conducting microscopy and their technical support. We thank Mr William Ellis at the Walter Reed Army Institute of Research (WRAIR) for providing reference drug supplies. We are appreciative of our colleagues at AFRIMS for their assistance: Ms Somporn Krasaesub for advice on statistical analysis, and Ms Tippa Wongstitwilairoong for assistance with preparation of illustrations and Figures. This work was funded by the Global Emerging Infections Surveillance (GEIS) Programme, US Armed Forces Health Surveillance Center (AFHSC), US Department of Defense.

\section{Competing interests}

The authors declare that they have no competing interests.

\section{Availability of data and materials}

The primary data are archived at the Armed Forces Research Institute of Medical Sciences. Once published they will be shared with the Worldwide Antimalarial Research Network (WWARN) repository.

\section{Consent for publication}

All study subjects agreed to publication of the study results in the medical literature without their identity at consent prior to participation.

\section{Disclaimer}

The views expressed in this article are those of the authors and do not reflect the official policy of the US Department of the Army, US Department of Defense, or the US Governments.

\section{Ethics approval and consent to participate}

All protocols were approved by the Walter Reed Army Institute of Research (WRAIR) Institutional Review Board and Cambodian National Ethics Committee for Health Research (NECHR), Institute for Development of Human Research Protection (IHRP), Ministry of Public Health, Thailand or Royal Thai Army Institutional Review Board (RTA IRB), respectively. Protocol approval reference numbers include WR1576 (minimal risk), WR1917 (minimal risk), WR1877 (NCT01849640), WR1737 (NCT01280162) and WR2017 (NCT02052323). All study subjects provided informed consent prior to participation.

\section{Funding}

Funding for the study was provided by the US Armed Forces Health Surveillance Center. The funding source did not participate in data analysis or the final decision to publish the manuscript.

Received: 15 June 2016 Accepted: 7 October 2016 Published online: 21 October 2016

\section{References}

1. WHO. World malaria report 2015. Geneva: World Health Organization; 2015.

2. WHO. Guidelines for the treatment of malaria. Geneva: World Health Organization; 2015.

3. Dondorp AM, Nosten F, Yi P, Das D, Phyo AP, Tarning J, et al. Artemisinin resistance in Plasmodium falciparum malaria. N Engl J Med. 2009;361:455-67.

4. Noedl H, Se Y, Schaecher K, Smith BL, Socheat D, Fukuda MM, et al. Evidence of artemisinin-resistant malaria in western Cambodia. N Engl J Med. 2008;359:2619-20.
5. WHO. Status report on artemisinin resistance. Geneva: World Health Organization; 2016

6. Ashley EA, Dhorda M, Fairhurst RM, Amaratunga C, Lim P, Suon S, et al. Spread of artemisinin resistance in Plasmodium falciparum malaria. N Engl J Med. 2014;371:411-23.

7. Denis MB, Tsuyuoka R, Poravuth Y, Narann TS, Seila S, Lim C, et al. Surveillance of the efficacy of artesunate and mefloquine combination for the treatment of uncomplicated falciparum malaria in Cambodia. Trop Med Int Health. 2006:11:1360-6.

8. Hung TY, Davis TM, llett KF, Karunajeewa H, Hewitt S, Denis MB, et al. Population pharmacokinetics of piperaquine in adults and children with uncomplicated falciparum or vivax malaria. Br J Clin Pharmacol. 2004;57:253-62.

9. Karunajeewa H, Lim C, Hung TY, Ilett KF, Denis MB, Socheat D, et al. Safety evaluation of fixed combination piperaquine plus dihydroartemisinin (Artekin) in Cambodian children and adults with malaria. Br J Clin Pharmacol. 2004;57:93-9.

10. Janssens B, van Herp M, Goubert L, Chan S, Uong S, Nong S, et al. A randomized open study to assess the efficacy and tolerability of dihydroartemisinin-piperaquine for the treatment of uncomplicated falciparum malaria in Cambodia. Trop Med Int Health. 2007;12:251-9.

11. Song J, Socheat D, Tan B, Seila S, Xu Y, Ou F, et al. Randomized trials of artemisinin-piperaquine, dihydroartemisinin-piperaquine phosphate and artemether-lumefantrine for the treatment of multi-drug resistant falciparum malaria in Cambodia-Thailand border area. Malar J. 2011;10:231.

12. Denis MB, Davis TM, Hewitt S, Incardona S, Nimol K, Fandeur T, et al. Efficacy and safety of dihydroartemisinin-piperaquine (Artekin) in Cambodian children and adults with uncomplicated falciparum malaria. Clin Infect Dis. 2002;35:1469-76.

13. WHO. National treatment guidelines for malaria in Cambodia. Geneva: World Health Organization; 2012.

14. Leang R, Barrette A, Bouth DM, Menard D, Abdur R, Duong S, et al. Efficacy of dihydroartemisinin-piperaquine for treatment of uncomplicated Plasmodium falciparum and Plasmodium vivax in Cambodia, 2008-2010. Antimicrob Agents Chemother. 2013;57:818-26.

15. Chaorattanakawee S, Saunders DL, Sea D, Chanarat N, Yingyuen K, Sundrakes $S$, et al. Ex vivo drug susceptibility testing and molecular profiling of clinical Plasmodium falciparum isolates from Cambodia from 2008 to 2013 suggest emerging piperaquine resistance. Antimicrob Agents Chemother. 2015:59:4631-43.

16. Saunders DL, Vanachayangkul P, Lon C. Dihydroartemisinin-piperaquine failure in Cambodia. N Engl J Med. 2014;371:484-5.

17. Spring MD, Lin JT, Manning JE, Vanachayangkul P, Somethy S, Bun R, et al. Dihydroartemisinin-piperaquine failure associated with a triple mutant including kelch13 C580Y in Cambodia: an observational cohort study. Lancet Infect Dis. 2015;15:683-91.

18. Amaratunga C, Lim P, Suon S, Sreng S, Mao S, Sopha C, et al. Dihydroartemisinin-piperaquine resistance in Plasmodium falciparum malaria in Cambodia: a multisite prospective cohort study. Lancet Infect Dis. 2016;16:357-65.

19. Leang R, Taylor WR, Bouth DM, Song L, Tarning J, Char MC, et al. Evidence of Plasmodium falciparum malaria multidrug resistance to artemisinin and piperaquine in Western Cambodia: dihydroartemisinin-piperaquine open-label multicenter clinical Assessment. Antimicrob Agents Chemother. 2015:59:4719-26.

20. Lim P, Dek D, Try V, Sreng S, Suon S, Fairhurst RM. Decreasing pfmdr1 copy number suggests that Plasmodium falciparum in Western Cambodia is regaining in vitro susceptibility to mefloquine. Antimicrob Agents Chemother. 2015;59:2934-7.

21. Saunders D, Lon C. Combination therapies for malaria are failing-what next? Lancet Infect Dis. 2016;16:274-5.

22. Chaorattanakawee S, Lanteri CA, Sundrakes S, Yingyuen K, Gosi P, Chanarat $\mathrm{N}$, et al. Attenuation of Plasmodium falciparum in vitro drug resistance phenotype following culture adaptation compared to fresh clinical isolates in Cambodia. Malar J. 2015;14:486.

23. Chaorattanakawee S, Tyner SD, Lon C, Yingyuen K, Ruttvisutinunt W, Sundrakes $S$, et al. Direct comparison of the histidine-rich protein-2 enzyme-linked immunosorbent assay (HRP-2 ELISA) and malaria SYBR green I fluorescence (MSF) drug sensitivity tests in Plasmodium falciparum reference clones and fresh ex vivo field isolates from Cambodia. Malar J. 2013;12:239. 
24. Saunders DL, Chaorattanakawee S, Gosi P, Lanteri C, Somethy S, Kuntawunginn W, et al. Atovaquone-proguanil remains a potential stopgap therapy for multidrug-resistant Plasmodium falciparum in areas along the Thai-Cambodian border. Antimicrob Agents Chemother. 2015;60:1896-8.

25. Witkowski B, Amaratunga C, Khim N, Sreng S, Chim P, Kim S, et al. Novel phenotypic assays for the detection of artemisinin-resistant Plasmodium falciparum malaria in Cambodia: in vitro and ex vivo drug-response studies. Lancet Infect Dis. 2013;13:1043-9.

26. Duru V, Khim N, Leang R, Kim S, Domergue A, Kloeung N, et al. Plasmodium falciparum dihydroartemisinin-piperaquine failures in Cambodia are associated with mutant K13 parasites presenting high survival rates in novel piperaquine in vitro assays: retrospective and prospective investigations. BMC Med. 2015;13:305.

27. Beshir KB, Sutherland CJ, Sawa P, Drakeley CJ, Okell L, Mweresa CK, et al. Residual Plasmodium falciparum parasitemia in Kenyan children after artemisinin-combination therapy is associated with increased transmission to mosquitoes and parasite recurrence. J Infect Dis. 2013:208:2017-24.

28. Lon C, Manning JE, Vanachayangkul P, So M, Sea D, Se Y, et al. Efficacy of two versus three-day regimens of dihydroartemisinin-piperaquine for uncomplicated malaria in military personnel in northern Cambodia: an open-label randomized trial. PLoS ONE. 2014;9:e93138.

29. Tarning J, Ashley EA, Lindegardh N, Stepniewska K, Phaiphun L, Day NP, et al. Population pharmacokinetics of piperaquine after two different treatment regimens with dihydroartemisinin-piperaquine in patients with Plasmodium falciparum malaria in Thailand. Antimicrob Agents Chemother. 2008;52:1052-61.
30. Woodrow CJ, Dahlstrom S, Cooksey R, Flegg JA, Le Nagard H, Mentre F, et al. High-throughput analysis of antimalarial susceptibility data by the WorldWide Antimalarial Resistance Network (WWARN) in vitro analysis and reporting tool. Antimicrob Agents Chemother. 2013;57:3121-30.

31. Maude RJ, Nguon C, Dondorp AM, White LJ, White NJ. The diminishing returns of atovaquone-proguanil for elimination of Plasmodium falciparum malaria: modelling mass drug administration and treatment. Malar J. 2014; 13:380.

32. Musset L, Bouchaud O, Matheron S, Massias L, Le Bras J. Clinical atovaquone-proguanil resistance of Plasmodium falciparum associated with cytochrome b codon 268 mutations. Microbes Infect. 2006:8:2599-604

33. Sutherland CJ, Laundy M, Price N, Burke M, Fivelman QL, Pasvol G, et al. Mutations in the Plasmodium falciparum cytochrome b gene are associated with delayed parasite recrudescence in malaria patients treated with atovaquone-proguanil. Malar J. 2008;7:240.

34. White NJ, Pukrittayakamee S, Phyo AP, Rueangweerayut R, Nosten F, Jittamala $\mathrm{P}$, et al. Spiroindolone KAE609 for falciparum and vivax malaria. N Engl J Med. 2014;371:403-10.

35. Darpo B, Ferber G, Siegl P, Laurijssens B, Macintyre F, Toovey S, et al. Evaluation of the QT effect of a combination of piperaquine and a novel anti-malarial drug candidate OZ439, for the treatment of uncomplicated malaria. Br J Clin Pharmacol. 2015;80:706-15.

\section{Submit your next manuscript to BioMed Central and we will help you at every step:}

- We accept pre-submission inquiries

- Our selector tool helps you to find the most relevant journal

- We provide round the clock customer support

- Convenient online submission

- Thorough peer review

- Inclusion in PubMed and all major indexing services

- Maximum visibility for your research

Submit your manuscript at www.biomedcentral.com/submit
() Biomed Central 